\title{
PULSIONS ET DESTINS DE PULSIONS - BREVE INTRODUCTION A CARMELO BENE
}

\author{
Jacques Aumont ${ }^{1}$
}

\begin{abstract}
Resumo: Ator e homem de teatro, escritor e cineasta, Carmelo Bene causou sensação desde o seu primeiro filme Notre-Dame des Turcs (1968). Inspirado em seu romance inaugural - uma espécie de autobiografia, mais propriamente poética que romanceada, ele permanece como sendo sua obra a mais célebre, verdadeiro turbilhão de imagens musicais, com seus monólogos, suas cores suntuosas, seu barroquismo simbolista. Eis por que Bene foi bastante admirado pelos críticos franceses, Deleuze, Klossowski e vários outros. Se seu cinema não tem um estilo reconhecível de um filme a outro, é verdade, no entanto, que, em todos os seus filmes, desde Notre-Dame des Turcs, ele pratica uma montagem disjuntiva que não se assemelha a nada do mesmo gênero. O cinema de Bene é esse exercício que só pode se receber por meio de um exercício igual da atenção, da tensão.

Palavras-Chave: Carmelo Bene; Teatro; Literatura; Cinema; Montagem disjuntiva.
\end{abstract}

Résumé: Acteur puis homme de théâtre, écrivain et cinéaste, Carmelo Bene a créé la sensation dès son premier film Notre-Dame des Turcs (1968). Inspiré de son roman inaugural - une espèce d'autobiographie, poétisée plutôt que romancée - il reste son œuvre la plus célèbre, véritable torrent d'images musicales, avec ses monologues, ses couleurs somptueuses, son baroquisme symboliste. C'est pourquoi Bene a été admiré par les critiques français, Deleuze, Klossowski et bien d'autres. Si son cinéma n'a pas un style exportable d'un film au suivant, ce qui est vrai en revanche, c'est que, dans tous ses films et dès Notre-Dame des Turcs, il pratique un montage disjonctif qui ne ressemble à rien du même genre. Le cinéma de Bene, c'est cet exercice pulsionnel qui ne peut se recevoir que par un exercice égal de l'attention, de la tension.

Mots-Clés: Carmelo Bene; Théâtre; Littérature; Cinéma; Montage disjonctif.

Septembre 1968. Les ondes de Mai n'en finissent pas de se propager, avec leurs hauts d'espoir et leurs bas de bêtise. Un jeune critique des Cahiers du cinéma, envoyé par son journal au festival de Venise, reçoit dans

1 Professor da Universidade de Paris III - Sorbonne Nouvelle, responsável por uma série de estudos sobre o cinema, em vasta bibliografia; dentre outras, é autor das obras: $A$ estética do filme (1995), Notre-Dame des Turcs: Carmelo Bene, 1968 (2010). Endereço eletrônico: jacques.aumont@ehess.fr. 
un grand désordre émotionnel un Bertolucci tonique et poétique (Partner), un Pasolini théorique et fantastique (Théorème), et les premiers films de deux inconnus, Maurice Pialat (L'Enfance nue) et Carmelo Bene. On me permettra d'avouer à la première personne que je n'avais rien compris à I'un ni à l'autre de ces deux derniers films. Devant Notre-Dame des Turcs, d'ailleurs projeté sans sous-titres, je mesurais mon inculture et les limites de la politique des auteurs. Je n'étais pas prêt pour ce torrent d'images musicales, ces monologues lyriques et sarcastiques, ces couleurs somptueuses et hideuses, ce baroquisme symboliste. Je ne savais rien de Bene, de sa réputation sulfureuse et prestigieuse. J'ignorais qu'il était un des plus remarquables hommes de théâtre de l'après-guerre, et qu'à sa carrière de dramaturge et d'acteur il venait d'ajouter celles de l'écrivain et du cinéaste.

À vingt-six ans, en 64, Bene avait arrêté subitement le théâtre. Réfugié avec sa compagne Lydia Mancinelli dans la maison de son père à Santa Cesarea Terme, au sud du talon de la botte italienne, il avait écrit un premier roman, Notre-Dame des Turcs. II écrivait lentement, récitant son texte à voix haute et à mesure, en homme de théâtre invétéré, fumant, buvant le café de sa mère et le rosé de son père, et dormant le moins possible. Ce livre sortait de lui sur le mode le plus banalement créatif, comme la pomme du pommier, comme l'enfant du ventre de la femme. Luimême dira: "Dès les premières pages, j'étais sûr de cracher un grand livre ". Ce n'est d'ailleurs pas un roman mais une autobiographie, poétisée plutôt que romancée. Une autobiographie sans dates, sans noms propres, sans anecdotes, mais qui dit tout de ce jeune homme alcoolique, mauvais coucheur, au charme immédiat et irrésistible, qui mettait dans sa poche les filles, les garçons, les directeurs de théâtre, les snobs et les malades mentaux (durant son bref séjour à l'asile de fous, il eut le temps de devenir dieu : ils le prenaient pour Allah ; on retrouve cet épisode dans le roman et dans le film). Notre-Dame des Turcs ne raconte pas des événements, mais une vie mentale proliférante : goût et dégoût des femmes; certitude d'être à part et dilatation de l'ego; fascination pour l'extase ; appartenance à un sud profond, oriental, " turc ".

À Venise, le film fit scandale. À l'époque, tout ce que faisait Bene aboutissait au scandale. Malgré leur inculture égale à la mienne, les critiques français, flattés que Bene leur eût réservé sa conférence de presse (dont il avait chassé les Italiens), en firent des comptes rendus assez éberlués pour paraître élogieux. En Italie le film ne parvint jamais à avoir une sortie décente; on parle encore de la dévastation de la salle Gioiello à 
Turin par des spectateurs mis en rage. En France, s'il n'eut pas de succès public, il fit événement. II inaugura un amour durable des milieux culturels et intellectuels français pour Bene, amour qui allait culminer dix ans plus tard avec les représentations de Richard III et les textes de Deleuze, de Klossowski et bien d'autres.

Carmelo Bene n'était pas écrivain ; il n'avait jamais appris à écrire. Je ne peux m'empêcher de penser au "je n'ai jamais appris à dessiner » d'Eisenstein - un homme que Bene admirait, comme un avant-gardiste vivant admire un avant-gardiste mort. Eisenstein avait passé sa vie à esquisser, sur tous les supports, les dessins les plus extravagants; Bene griffonna toute sa vie des bouts de "romans ", des textes d'autobiographie et d'autojustification, des poèmes, des scénarios, des livrets pour le théâtre (on ne peut pas appeler ça des pièces), sans parler des lettres aux amis et aux femmes. Toujours, devant ces écrits, une unique sensation, une unique certitude - celle d'une urgence qui serait totalement maîtrisée. Bene ne rédige pas traditionnellement, par chapitres, mais ses récits et ses essais ne sont pas erratiques, ils ont une structure, une grande forme. Cependant, à l'intérieur de cette grande forme, son style est infiniment varié. II peut accumuler les phrases de deux ou trois mots, ponctuées à la mitraillette. II peut aussi battre des records proustiens. II n'a pas de style fixe : l'écriture relève de la nécessité. Aussi son unité d'écriture n'est-elle ni le chapitre, ni la phrase, mais le paragraphe.

Je me rappelle, encore en France et encore dans l'après-68, le choc d'Eden, Eden, Eden, de Pierre Guyotat. Là aussi, urgence et maîtrise; violence et "pornographie ", ce qui lui valut la censure donc la gloire. Mais je me rappelle surtout, de Guyotat, sa défense de l'« écriture de phrase »: son livre n'était qu'une gigantesque phrase, pliée, tordue, forgée, martelée, laminée, tréfilée. Bene, non : c'est le paragraphe. Les romans de Bene enchaînent des paragraphes, ou plutôt les font se succéder par des cuts soudains. On comprend qu'il n'ait eu aucun mal à passer au cinéma : le montage, il l'avait déjà découvert et pratiqué dans l'écriture. Je relis n'importe quelle page de Notre-Dame des Turcs (dans la belle traduction de J.-P. Manganaro ou, si je suis en forme, en italien). Ou un chapitre de Je suis apparu à la Madone (il y en a au moins un traduit en français, pas trop mal). Ou mieux (mais pas traduit) L'orecchio mancante, par exemple le texte intitulé La Signora Felicità. À chaque fois je suis sidéré par ce règne de l'asyndète et de l'anacoluthe; d'un paragraphe au suivant, je me vois sommé de me réajuster mentalement, de tout recommencer, pourtant sans 
oublier rien de ce qui précède, et qui sera supposé. Ce n'est pas une accumulation décousue; pas non plus des éclairages différents sur une même réalité, fût-elle imaginaire. C'est un propos suivi, et même ordonné, mais son ordre ressortit à une logique non linéaire, jouant à plein de l'étoilement et de la dissémination du sens et des sensations, jouant sans limites du principe de montage.

La langue de Bene (je le dis avec la modestie qui convient à un italianiste amateur) est par endroits aussi inventive et aussi opaque que celle de Gadda. On y sent, comme chez Pasolini (un de ses rares contemporains que Bene ne trouvait pas nul) une familiarité profonde avec toute l'histoire de la langue italienne, depuis son origine mythique à la cour sicilienne de l'empereur allemand Frédéric II, par exemple la Rosa fresca aulentissima de Cielo d'Alcamo (un siècle et demi avant Dante). D'ailleurs je ne connais cela que parce que Bene a si bien expliqué, et si obsessionnellement, comment le plus profond de la culture littéraire italienne vient de ce sud du sud. C'est clair, il cultive ses mots avec un soin jaloux - pas seulement les adjectifs - , et chacun vaut pour sa résonance, émotionnelle et sensorielle. Il y a une volupté du mot que l'on savoure et déguste, un côté profondément esthète (Des Esseintes est un de ses modèles avoués). Cependant il n'est pas Flaubert, qui pouvait passer une journée sur une virgule. Ses phrases jaillissent, comme des vagues. Chaque paragraphe nous mène en barque, nous ballotte sur ces vagues. Jamais facile, avec tout ça, de savoir bien quel est l'itinéraire, et s'il y a un terme au voyage. Pourtant, cela avance.

Bene n'était pas davantage cinéaste qu'écrivain. II n'a cessé de vilipender le cinéma, ronchonnant « qu'on ne pouvait pas faire des films avec du cinéma ". De fait, ses films ne sont pas faits en cinéma, si le cinéma est cette institution floue mais puissante qui marie les produits de masse avec l' " art et essai » et le divertissement avec l' " expérimental ». Bene, dont la culture cinéphilique ne valait pas sa vaste et précise culture littéraire, avait tout de même bien perçu que, s'improvisant cinéaste, il ne voulait devenir ni Germi, ni Antonioni, ni Paolo Gioli (pour ne citer que des Italiens et ses contemporains). II ne ferait pas des succès populaires, pas des films d'art, pas du cinéma expérimental. Son cinéma n'a pas de modèle, et, je crois bien, n'a subi aucune influence. D'ailleurs, pas de style, en tout cas pas un style exportable d'un film au suivant, et qui ferait marque de fabrique ou marque autorale. Bene s'est souvent vanté, et félicité, du montage serré de ses films, mais ce n'est littéralement vrai que de Salomé 
(1972), dont les plans sont en effet très brefs. Ce qui est vrai en revanche, c'est que, dans tous ses films et dès Notre-Dame des Turcs (qui à mes yeux reste son chef-d'œuvre absolu), il pratique un montage disjonctif qui ne ressemble à rien du même genre, ni Eisenstein ni le Welles d'Othello, auxquels on l'a parfois comparé, ni Vertov auquel il serait davantage apparenté si on faisait abstraction d'idéologie, ni le Brakhage de la trilogie de Pittsburgh auquel pourtant cela parfois fait penser par la neutralité subjective (encore un oxymore) du cadrage.

Des films sans style - au sens "le style c'est l'homme " comme au sens de la soumission à une norme. Des films qui n'entrent dans aucune catégorie constituée de l'art cinématographique. Avec chacun de ses cinq longs-métrages, manifestement Bene veut faire du neuf, à tous les points de vue. Je repense à la fameuse phrase de Benjamin, postulant que les grandes œuvres sont celles qui inventent leur genre, ou le détruisent. Aucun de ces cinq films ne ressortit à un genre constitué : Notre-Dame des Turcs n'est ni de la comédie, ni de l'autofiction (genre d'ailleurs inexistant en 1968) ni un journal filmé (comme le faisait Mekas); Bene y invente le genre de l'autobiographie mentale, un récit de vie qui ne raconte rien mais dise tout. L'autre chef-d'œuvre de Bene, Salomé, n'est pas une adaptation théâtrale, quoique le texte y soit, mais coupé, allongé, remonté avec celui d'une autre pièce, rajoutant de l'ironie à l'ironie de Wilde; ce n'est pas non plus de l'underground, malgré le montage ultracourt; là aussi, un genre s'invente, quelque chose comme un opéra poétique sans musique. Même Un Amleto di meno, qui est le moins séduisant de tous, propose une posture neuve entre le cinéma et le théâtre, celle d'une fausse répétition dans les vrais costumes, une " couturière " sans scénographie. De même Capricci est trompeur si l'on n'y voit qu'un remake ou une parodie de Week-end, rapprochement qui avait le don d'agacer prodigieusement le grand Carmelo ; ce dont il est le plus proche narrativement, ce sont les deux petits contes de Pasolini, La Terra vista dalla Luna et Che cosa sono le nuvole?, pour leur fantaisie et leur digressions - mais évidemment en plus gros, en plus délibérément vulgaire. À chaque fois, l'invention d'un genre, et en prime, la destruction immédiate de ce genre à peine constitué.

Un amateur en littérature et en cinéma, et qui sciemment refuse toutes les avenues balisées pour frayer un chemin inexploré, et le frayer également par le mot et par l'image mouvante. Comme si écrire et filmer, pour lui c'était la même chose. Mise en jeu de la pulsion, et comment : tout part de là, tout est là, il n'y a rien d'autre, tout est pulsion dans le texte ou 
le film ; les phrases emballent notre lecture parce qu'elles ont été écrites à voix haute; les plans nous sidèrent et nous choquent parce que le cinéaste et comédien s'y livre entièrement, au risque de tout le corps. Et cependant contrôle, et même contrôle méticuleux, et même, maniaque. Dans les textes, le sentiment qu'un adverbe a été pesé longuement, préféré à douze autres ou à aucun; pas Flaubert et ses virgules, plutôt Huysmans ou Laforgue (deux références expresses de C. B.) et leur goût décadentiste pour la richesse de la prose. Dans les films, l'évidence d'un cadrage toujours étonnamment plein, d'une image sensuelle et en même temps économique, sans gras. À chaque re-vision je suis ébloui par la science innée des cadres, y compris l'art de maîtriser leur durée au plus juste, chez cet autodidacte. En vieillissant, Bene figera ce double personnage d'amateur pulsionnel, doté d'une vitalité et d'une créativité hors normes, et de monstre de culture et de précision de l'intellect. Le sommet est atteint avec la Vita di Carmelo Bene, long entretien autobiographique avec le journaliste Giancarlo Dotti, un spécialiste du foot et assistant occasionnel du maître : sur la spontanéité et les digressions de l'oral, Bene a pour la publication en livre greffé de longs passages théoriques, épatants, autoritaires - et chantournés à l'excès.

Carmelo Bene n'était pas un écrivain, il n'était pas un cinéaste. S'il était quelque chose, c'était, intensément, passionnément, absolument, un homme de théâtre. Un homme de théâtre, et pas, par exemple, un acteur ou un dramaturge ou un metteur en scène. II vomissait l'idée même de mise en scène, ne reconnaissait qu'une forme de théâtre, fondée sur ce qu'il dénommait "écriture scénique ", c'est-à-dire sur la mise en jeu et en danger permanent du corps de l'acteur sur la scène, et donc, forcément, sur une bonne dose d'improvisation ou, plus justement, de présence. Pas un écrivain professionnel, pas un cinéaste professionnel. Au vrai, professionnel, amateur, ces mots pour lui n'avaient aucun sens. II ne reconnaissait à sa vie qu'un seul centre, un seul principe ordonnateur : le don entier, permanent et irrévocable de sa personne au théâtre (" être sur scène ou ne pas être " : on lui prête ce mot, lui qui à vingt ans se vantait de connaître par cœur une dizaine de traductions différentes de Hamlet).

Écrire, filmer : toujours pour faire passer quelque chose de l'ordre du corps. Le corps est, pour ce successeur d'Artaud, la seule réalité dont nous puissions être raisonnablement certains, y compris dans ses insuffisances : comme son grand ancêtre, Bene aurait bien voulu " faire son corps luimême "; il s'y appliqua en le ruinant systématiquement. La relation entre 
cinéma et littérature n'est alors plus à penser d'aucune des manières habituelles. Pas d'“ adaptation " entre littérature, cinéma, théâtre ; seulement une écriture pulsionnelle et incessante, qui seulement change de vêtement. Aucune écriture sinon celle où le corps peut s'investir : écrire c'est jouer, écrire c'est vivre. Pas de récit mais des tresses de monologues intérieurs, d'où émerge quelque chose comme une histoire, mais toujours incertaine et laissée à ma collaboration. Pas de personnage, mais des psychismes incessamment dissociés, auxquels il n'est plus question de s'identifier. Ni la littérature, ni le cinéma ne sortent intacts de cette prodigieuse machine actorale. Bene parlait, à propos du roman Notre-Dame des Turcs, de "parodie affolée de monologue intérieur" : ce qui compte dans cette formule, c'est moins le monologue intérieur et même sa parodie que l' « affolement ». Le monologue intérieur, bien sûr, avait ses lettres de noblesse pour Bene, qui admirait aussi cet autre avant-gardiste, Joyce ; la parodie, sans doute, lui est consubstantielle, il ne sait rien faire que ce " chant à côté ". Mais sa marque, c'est l'affolement : la vitesse, la nervosité, le devenir-fou (au sens psychique comme au sens mécanique).

En 1979, à l'orée de sa réputation de grand poète et de grand intellectuel, Bene écrit avec Deleuze, non à quatre mains mais en surimpression (en Superpositions, selon le très exact titre du livre), un essai sur sa pratique du théâtre et du cinéma. Le philosophe, qui entre-temps a découvert pour son compte propre les films et les mises en scène de Bene, en donne une version adaptée à ses propres obsessions du moment : la littérature mineure et le minoritaire en général, la répétition et la variation opposées au développement linéaire (le côté wildien de Bene), l'ambiguïté et le travesti, l'intensivité, la machine plutôt que l'appareil. Ce n'est pas mal vu, et ce texte reste l'un des plus denses et des plus lucides sur la situation du poète italien à ce moment-là ; pourtant j'ai toujours envie de lire Bene, plutôt, à la lumière d'une autre idée de Deleuze, proposée celle-là à propos de Beckett.

Le héros de Notre-Dame des Turcs, pour ne prendre que lui (mais on pourrait le dire du personnage de Bene dans Capricci, du Hérode de Salomé et même de Don Giovanni) est ce personnage de fiction à qui il n'arrive rien sauf d'innombrables aventures mentales (définition qui, au fond, irait aussi à Hamlet). La conception benienne du personnage, c'est de recréer un corps à partir de ce qu'il est, en le confrontant à des forces rien moins que vitales - de laisser couler à travers ce corps le flux de la vie. Or ce passage incessant, cette soumission à une énergie, à une force supérieure, tout 
simplement l'épuise. Le personnage est traversé par des forces vitales, et ça l'épuise : ce pourrait être aussi une définition du film, de tous les films de Bene, qui sont des explosions de force figurative laissant la forme épuisée (au sens, aussi, où tout a été exhaustivement essayé). Ce pourrait aussi être une description de la relation que ce cinéma suscite avec son spectateur : cette grande forme de l'épuisement nous vide, nous aussi, après avoir exigé notre adhésion entière. Le cinéma de Bene, c'est cet exercice pulsionnel qui ne peut se recevoir que par un exercice égal (de l'attention, de la tension), et qui fait de nous en miroir un épuisé : non pas un fatigué, mais quelqu'un qui a donné tout ce qu'il pouvait.

C'est dans L'Épuisé (de Deleuze) qu'on trouve cette définition: " L'image n'est pas une représentation d'objet, mais un mouvement dans le monde de l'esprit. " II importe assez peu, dès lors, que ce soit une image littéraire, une image filmique, une image théâtrale. L'art de Bene, c'est d'avoir tout ramené à la racine de l'idée d'image, de n'avoir jamais fait $a$ priori acception de médium - moyennant quoi il bouscule, renouvelle, viole tous les médiums, et en passant les exalte.

Dans l'espace d'un même paragraphe de la Vita di Carmelo Bene, on lit ces deux affirmations: "On ne lit pas pour juger les chefs-d'œuvre mais pour vérifier ce qu'on vaut. Je vaux un fameux rien du tout. " - et : "Chefd'œuvre ? II faut être un chef-d'œuvre. " Orgueil et humilité, également sincères. Mais par-delà l'apparente contradiction, elles disent la même chose : à l'aune du chef-d'œuvre je ne suis rien, mais je peux vouloir être tout, si je cesse de chercher le chef-d'œuvre dans l'œuvre pour le trouver dans son œuvrement, si je reconnais qu'il n'est d'œuvre que dans l'incessant mouvement de la vie. "En vérité, c'est la vie même. "

On peut être profondément original et ne pas constituer une origine : Bene n'a aucune postérité, en cinéma en tout cas et sans même parler de sa dernière période, à partir de la mémorable Lectura Dantis de 1981 à Bologne, qui fit de lui un homme public. Je ne vois personne qui ait su jouer tous les rôles et mettre en jeu comme lui l'incessant montage de la pulsion, par toutes les voies et sous toutes les formes à la fois. II y a eu, depuis, nombre de cinéastes qui ont bousculé le récit et poussé la fiction à sa limite, y compris dans l'industrie hollywoodienne, mais le seul, je crois bien, qui ait accompli une entreprise comparable, centrée sur l'acteur pour mieux le déposséder de tout rôle, c'est le premier Schroeter, c'est-à-dire un de ses contemporains (même Garrel n'a pas été aussi loin). Quant au corps et à sa mise en jeu, je suis frappé qu'aucun des hommes de théâtre qui 
sont, plus ou moins occasionnellement, passés au cinéma n'en ait manifesté le souci que pour aussitôt se défausser de toute pulsion en la déléguant à des acteurs professionnels (on le voit bien chez Chéreau). Les films de Bene ont été des aérolithes, pas une prophétie.

\section{RÉFÉRENCES}

AUMONT, Jacques. " Notre-Dame des Turcs » de Carmelo Bene. Lyon: Aléas, 2010.

BENE, Carmelo. Nostra signora dei Turchi. Trad. fr.: Notre-Dame des Turcs. Paris: POL, 2003.

BENE, Carmelo. L'orecchio mancante. In: Idem. Opere. Milano: Bompiani, 1995.

BENE, Carmelo; DOTTI, Giancarlo. Vita di Carmelo Bene. Milano: Bompiani, 1998.

DELEUZE, Gilles. L'Épuisé. In: BECKETT, Samuel. Quad et autres textes. Paris: Éd. de Minuit, 1992.

DELEUZE, Gilles; BENE, Carmelo. Superpositions. Paris: Éd. de Minuit, 1979.

GUYOTAT, Pierre. Éden, Éden, Éden. Paris: Gallimard, 1970.

LAFORGUE, Jules. Hamlet, ou les suites de la piété filiale [1887]. In: Idem. Moralités légendaires. Paris: Flammarion, 2000. 
